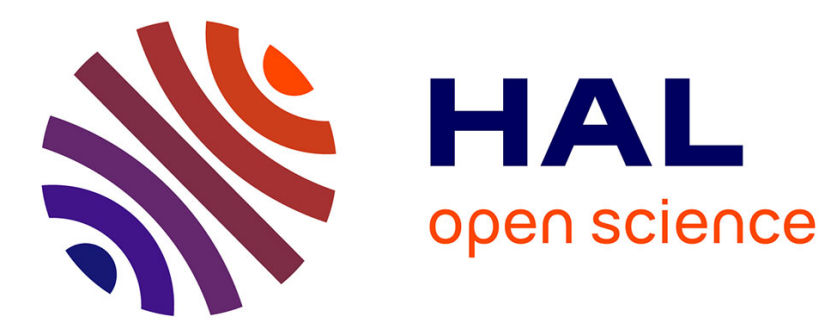

\title{
Topological characterization of mobile robot behavior
}

\author{
Aurélien Hazan, Frédéric Davesne, Vincent Vigneron, Hichem Maaref
}

\section{To cite this version:}

Aurélien Hazan, Frédéric Davesne, Vincent Vigneron, Hichem Maaref. Topological characterization of mobile robot behavior. International Conference on Intelligent Robots and Systems (IROS 2006), Oct 2006, Beijing, China. pp.4157-4162, 10.1109/IROS.2006.281905 . hal-00203675

\section{HAL Id: hal-00203675 \\ https://hal.science/hal-00203675}

Submitted on 10 Jan 2008

HAL is a multi-disciplinary open access archive for the deposit and dissemination of scientific research documents, whether they are published or not. The documents may come from teaching and research institutions in France or abroad, or from public or private research centers.
L'archive ouverte pluridisciplinaire HAL, est destinée au dépôt et à la diffusion de documents scientifiques de niveau recherche, publiés ou non, émanant des établissements d'enseignement et de recherche français ou étrangers, des laboratoires publics ou privés. 


\title{
Topological characterization of mobile robot behavior
}

\author{
Aurélien Hazan, Frédéric Davesne, Vincent Vigneron, Hichem Maaref \\ Image and Signal Processing Team \\ IBISC, FRE 2873 \\ 91020 Evry Cedex \\ Email: aurelien.hazan@ibisc.fr
}

\begin{abstract}
We propose to classify the behaviors of a mobile robot thanks to topological methods as an alternative to metric ones. To do so, we adapt an analysis scheme from Physics of nonlinear systems in chaotic regime, assuming a dissipative dynamics that relaxes on a low-dimensional manifold. Sensor data recorded from a mobile robot during a wall-following experiment allows to compute topological invariants that give a standardized representation of the structure of the set of trajectories, and enable us to discriminate among similar behaviors in a systematic and quantitative way.
\end{abstract}

\section{DESCRIBING THE BEHAVIOR OF A MOBILE ROBOT}

In this article we address the problem of classifying the behavior of a mobile robot, where by behavior, we mean how the robot interacts with its environment, not only where the robot is located. Whether they favor a deterministic or random treatment, most works addressing this topic in the literature adopt a metric stand, we shall see in what sense.

On one hand, when the phenomenon is modeled as a random process, as in [22] and [15], the first step consists in digitizing the signal so that the transition between a finite set of symbols can approximate the initial time series. Then, the time series is considered as a Markov Chain, i.e. the transition probability from one state $x(n)$ to another $x(n+1)$ depends only on the step $x(n)$. Under this assumption, one can estimate the transition probability from the transition frequence. Finally, the Markov Chains undergo a classification stage under some optimality constraint. An important detail is that this clustering is made on the basis of a similarity measure between probability distributions, e.g. the Kullback-Leibler distance in the case of [22]. Some works, such as [25], make no assumption on the nature -deterministic or random- of the underlying phenomenon, and apply data analysis techniques such as Self Organizing Maps to recorded sensor data, in order to classify different trajectories on a metric basis. In all cases those attempts to characterize the behavior of a robot rely on the knowledge of a distance.

On the other hand, when the phenomenon is modeled as a deterministic one, as in [3] and [23] in the cybernetic tradition dating back to Ashby, a functional relation links sensory and motor variables, in the form $\dot{x}=F(x, t, q)$, where $x$ is the state variable, $t$ the time, and $q$ a control parameter such as the nature of the environment. In that case, time series recorded from the robot are processed to compute metric and dynamical invariants such as Lyapunov exponents, correlation or fractal dimension as in [24], [20], [21] and [12]. Again, in that case, it is necessary to define a distance on the state space. In the next section we will argue that another standpoint, based on topological arguments, allows a sharp characterization of the set of trajectories.

\section{DYNAMICAL ANALYSIS}

The nature of the studied system strongly orients the tools used to describe it: when dealing with linear systems, one merely needs to estimate the Fourier spectrum of a phenomenon, since it is preserved by changes in time or in excitation strength. When the system is no longer linear, and happens to display chaotic behavior, the Fourier spectrum is no longer sufficient to characterize the dynamics. As proved by experiments in section IV (see also [20]) even a simple wallfollowing robot acts in a chaotic way, that's why we focus on tools able to cope both with regular and chaotic phenomena.

\section{A. Classical use of Nonlinear Dynamics tools}

Nonlinear Dynamics mixes the theory of Dynamical Systems, Control theory and Time series analysis and provides us with valuable tools to characterize the behavior of a system. Four types of invariance are sought by researcher when dealing with a means to characterize a system. The tool must be insensitive to:

1) evolution operator (i.e. it mustn't depend on the time interval for which the "invariants" are computed).

2) coordinate change, be it nonlinear (though smooth enough).

3) initial condition change.

4) noise.

Fractal dimensions and Lyapunov exponents show such invariance to points 1 to 3 (see [1], chapter 5, for a discussion), and suffer several numerical limitations including sensitivity to noise contamination (see [7] for example). Furthermore, they are not invariant to control parameter changes (robot speed, environment, command law), even when the overall dynamics keeps the same properties.

Still, to the best of our knowledge, when a roboticist intends to characterize the behavior of a system with the help of such tools, he solely computes those metric invariants without 
questioning their pertinence, and neglects other valuable tools available in the field of nonlinear dynamical analysis.

\section{B. Topological approach}

Dating back to Poincaré, physicists have urged that an explanation of a dynamical phenomena be carried on focusing on unstable periodic orbits. Until now, scientists keep that aim in mind and come up with efficient tools for analysis (see [2], [18], [6], [9] for a recent example applied to many systems including lasers, chemistry and neural activity). First, the non-wandering set -composed of the points whose asymptotic position remains bounded- is being approximated. Then, the unstable periodic orbits are isolated -most probably surrogate periodic orbits, since true periodic orbits are very hard to localize experimentally. Then a symbolic coding of the continuous signal is proposed: at this point, this theory meets the preoccupations of many researcher in behavioral and adaptive robotics concerned with the anchoring of symbols in measurements. Indeed those symbols result from the dynamics itself, though the observer may fail to isolate them while building an inappropriate state space or mispartitioning that space (see [13]). Some theorems ensure the existence of partitions that completely captures the dynamics, which allows one to work directly on symbolic sequences. Finally, from the symbolic coding of periodic orbits, topological invariants that form the fingerprint of the phenomena are computed, and show invariance to a set of perturbations broader than metric and dynamical invariants such as Lyapunov exponents or fractal dimension discussed in II-A (see [9], chap. 9 for a discussion).

\section{EXTRACTING TOPOLOGICAL CUES FROM EXPERIMENTS}

Our goal here is to associate a unique fingerprint to the behavior of a robot from recorded data, with no model or equation available before the experiment. As we saw in II, such preoccupations have existed for a long time in physics, where general theoretical results from Dynamical System Theory, Time Series Analysis were melt with particular experiments in order to classify phenomena. In this section we enumerate briefly the different tools necessary to the study conducted in section IV.

First we build an embedding with several possible dimensions, in three classical coordinate systems (time delay, differential-integral and SVD embeddings) in order to estimate the fittest embedding dimension $d_{E}$. By fittest we mean both lowest (in spite of what the embedding theorems could state since we need not a necessary but a sufficient result), and false-nearest-neighbor-free (see [16], [8]). For a general discussion on embedding and reconstruction of attractors in chaotic regime, see the classical work of Abarbanel in [1]. When these requirements are met, $d_{E}$ is frozen and no longer moves.

Secondly, we extract unstable periodic orbits thanks to a close return plot, that allows to isolate an interval of instants in time, and a period $p$ such that the distance between $x(t)$

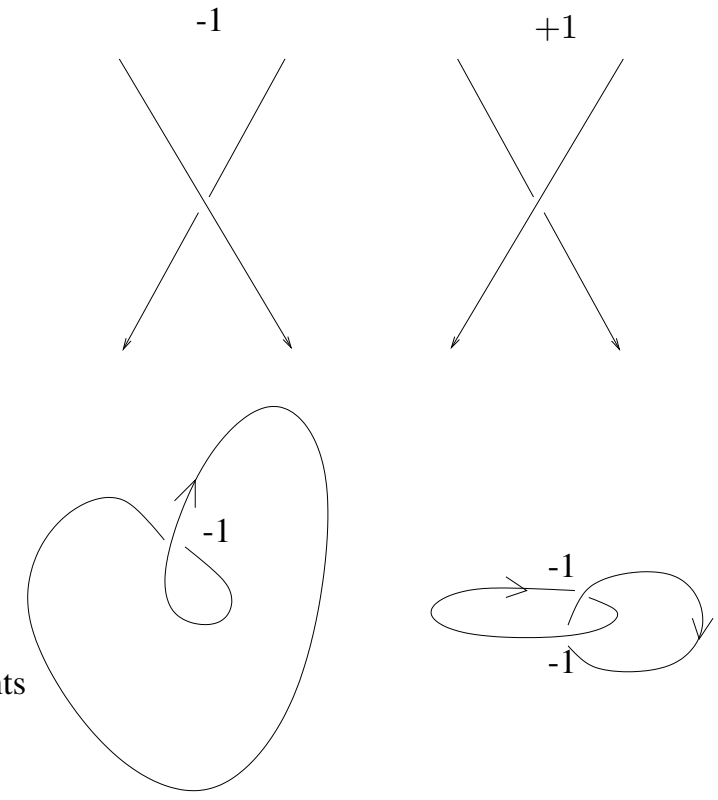

Fig. 1. Computation of topological invariants. (Top) Convention for counting signed crossings in projection on a $2 \mathrm{D}$ plane. (Bottom Left) Self-linking number of a periodic orbit, (Bottom Right) Linking Number of two periodic orbits.

and $x(t+p)$ remains lower than a threshold $\varepsilon$ typically smaller than $1 \%$ of the largest diameter of the attractor (see [19]).

Then, we need a symbolic encoding of the state-space, obtained for example by intersecting the flow with a wellchosen Poincaré section transverse to it (for precisions see for example [4]). Then, several cases can occur, the simplest being if the set of points is disposed along a line, for example the coordinate $x$ that represents the scalar recorded data. One can build a return map that maps $x(n)$ onto $x(n+1)$, the next intersection of the flow with the section. From that return map, one may identify a set of symbols using classical techniques such as identifying critical points (see [9]).

Even if the unstable periodic orbits have not been given a symbolic name, it is possible to classify them: one can compute topological invariants that depicts the properties of crossings and relative rotation between the isolated periodic orbits. To do so, one counts the signed number of crossings of a periodic orbit with itself, and the signed number of crossings for all couples of available low-period orbits that have been identified thanks to the close return plot. After Gilmore and Lefranc in [9] we note $S L(A)$ the self linking number or periodic orbit $A$, defined as the sum of all signed crossings $\sigma_{i}$ of the orbit with itself: $S L(A)=\sum_{i} \sigma_{i}(A)$. Similarly we note $L(A, B)$ half the sum of the signed crossings $\sigma_{i}(A, B)$ between periodic orbits $A$ and $B: L(A, B)=1 / 2 \sum_{i} \sigma_{i}(A, B)$. See Fig. 1 for more explanation on how to compute the invariants.

Finally the combination of symbolic names and topological invariants provides us with a unique fingerprint of the set or periodic orbits that form the skeleton of the whole attractor, and hence of the behavior of the system. 


\section{EXPERIMENTS}

In the following sections, we successively depict the simulator, the selected robot, its control algorithm and the environment in which the simulation takes place. Then we present the result when applying the method presented in III.

\section{A. Simulator}

Player is an open source device server initially developed at USC Robotics Research Lab in 1999 that allows, when connected to a series of sensors and actuators, to control them over a TCP socket with a request/reply mechanism. Userdesigned clients then connect to the server from the network to communicate with the controlled set of device (see [11], [10]), allowing multiple simultaneous connections. Player was initially programmed to control real robots, but comes with two simulators that replace the real robot as the last link of the client $\rightarrow$ device server $\rightarrow$ real device chain, Stage and Gazebo. The former copes with large population of simulated $2 \mathrm{D}$ robots, while the latter aims at simulating accurately small populations of 3D robots. It relies on the ODE physics engine that simulates the kinetics and dynamics of a set of articulated bodies in the presence of gravity and friction forces. As Player, Gazebo is a server to which clients send control requests and that sends back the current state of the system. For a detailed presentation, see [17].

Player/Stage/Gazebo is actively maintained by a large open source community, its use being widespread in universities and research labs in the USA and Canada. However, the validity of simulation is assessed by few, empirical articles (see [11] section 2.2.2, which provides a bibliography). Furthermore, the question of the observation of chaos on a computer deserves careful attention that exceeds the limits of this short article (section 2.13 of the nonlinear FAQ at http://www.faqs.org/faqs/sci/nonlinear-faq/ includes some useful references).

\section{B. Robot, Command, Environment}

Simulated experiments involved a Pioneer 2-AT robot equipped with a laser range finder, controlled by the VFH algorithm so as to follow right walls, in the environment depicted by Fig 2, taken from [12].

As it comes implemented in Player, we used VFH (vector field histogram), an obstacle avoidance algorithm that continuously builds a two-dimensional Cartesian histogram grid based on range sensors data. First, that histogram is projected in a polar coordinate system and stands for the obstacle density of presence in a given direction. Then, the region with lowest density is selected, and the robot is aimed at it. Since this algorithm is classical, we detail it no longer and refer the reader to [5].

\section{Results in the low speed case}

In this section we set the maximum robot speed to $v_{\max }=$

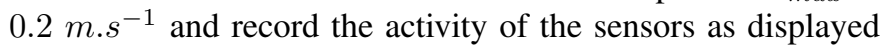
by Fig. 3(a). The system seems to be in periodic regime, its period being enclosed between arrows. In this section

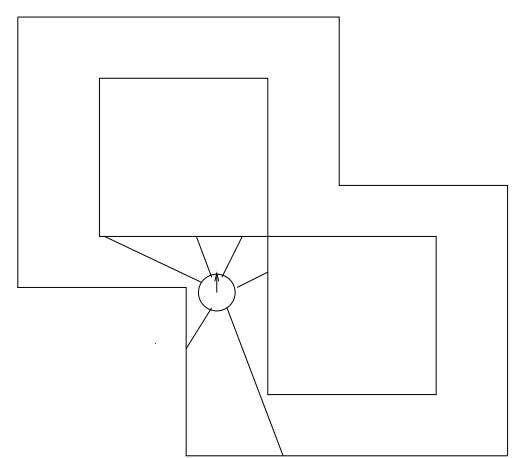

Fig. 2. Pioneer robot equipped with laser range-finder in maze environment.

and in the following, we study the signal recorded by one range sensor only, and after checking that the attractor was embeddable in dimension $d_{E}=3$ thanks to the false nearest neighbors algorithm, we embed it with the differential-integral coordinate system as can be seen in Fig. 3(b). Although the regime looked periodic, a careful examination reveals stretch or squeeze between branches, as evidenced by the squared area in Fig. 3(b), that are typical of chaotic regimes (see [9]). However, we prefer to study more carefully a case where the chaotic behavior is made even clearer in the recorded time series itself, which will be done in the next section.

\section{Results in the high speed case}

In this section we set the maximum robot speed to $v_{\max }=$ $0.4 \mathrm{~m} . \mathrm{s}^{-1}$, and record the activity of the sensors (see Fig. 4(a)). From just one scalar data taken from a particular sensor, we first evaluate in Fig. 4(b) the embedding dimension for time-delay embedding thanks to the false nearest neighbors algorithm, and establish that dimension $d_{E}=3$ will be convenient, despite a residual percentage of false neighbors due to noise. However, this embedding concentrates the orbits too tightly in some part of the phase space, which may hinder the computation of topological invariants. Instead, we embed the data in differential-integral coordinates as shown by Fig. 5(a). Since the important features of this reconstructed attractor, such as the number of branches, or the stretch and squeeze between branches are hard to perceive, we examine the intersection between the flow and a Poincaré section placed as shown by Fig. 5(b). From the computed section one can define a return map that maps each intersection with the next intersection after one cycle around the attractor. More specifically, we compute a one-dimensional return map that maps the $x$ coordinate of an intersection and the next $x$ coordinate. Fig. 5(c) displays the result, for the plane drawn on Fig. 5(b). We added the grid that separates arbitrarily the different branches composing the attractor in the region of the Poincaré section, so as to establish the allowed and forbidden sequences among branches. Here we meet the problem of partitioning the state space into symbols, as evoked earlier in sections I and II-B. Five symbols numbered from 0 to 4 are identified from the return map and from the study of patterns of the time series recorded in Fig. 4(a), their incidence matrix 


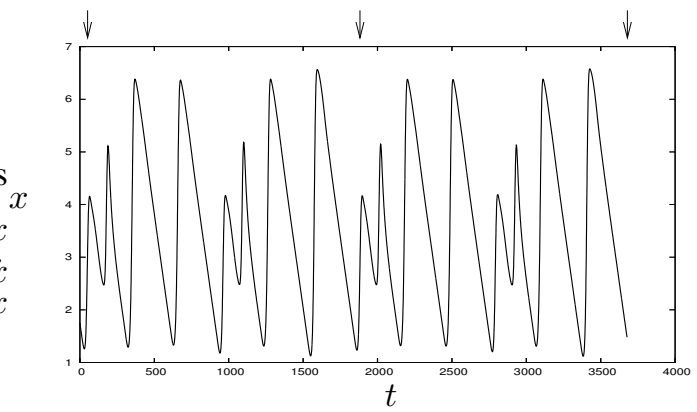

(a)

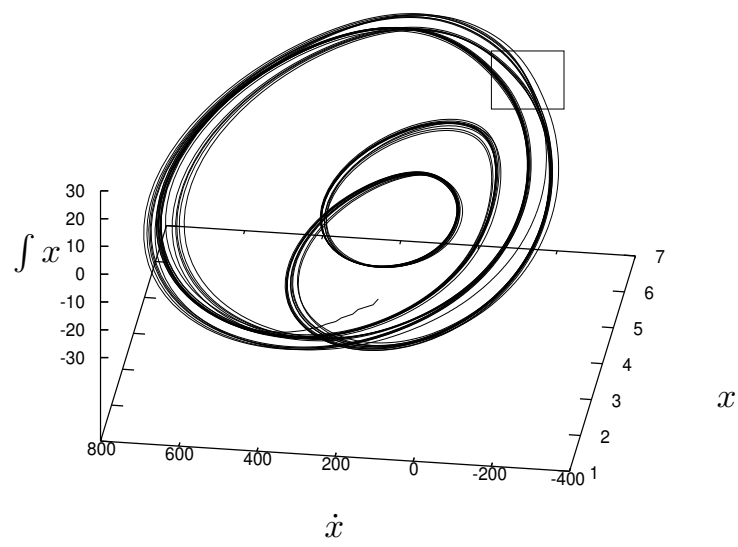

(b)

Fig. 3. Low-speed case. (a) Recorded activity of the third sensor -a period lies between two arrows. (b) Reconstructed attractor in differential-integral embedding.

being given by Table I. It reads as follows: orbit number $i$ in the first row can be followed by the orbit $j$ whose transition value $\left(t_{i j}\right)$ in the matrix is 1 . In some cases, it is not clear whether or not the transition is really allowed, since only one or two points belong to the box, among more than 400 extracted from the initial 65536. In those limit cases, we take the stand to neglect those points that might come from a mispartitioning of space or from outliers, and use bold font to remind of that choice.

Until now, the information gathered fails to be a $1: 1$ representation of the attractor, because it lacks its topological properties. They can be revealed by observing the unstable periodic orbits of the dynamics, more specifically their crossings, possibly quantified by the linking number and self-linking numbers between two trajectories. We isolate two periodic orbits, for example $0 \rightarrow 2 \rightarrow 3$, and $0 \rightarrow 1 \rightarrow 4$ and compute their topological invariants such as self-linking numbers for a single periodic orbit, or linking numbers between two orbits. Fig. 6 shows a projection of periodic orbit $0 \rightarrow$
TABLE I

INCIDENCE MATRIX, HIGH SPEED CASE

\begin{tabular}{|l||l|l|l|l|l|}
\hline & 0 & 1 & 2 & 3 & 4 \\
\hline \hline 0 & 0 & 1 & 1 & 0 & 0 \\
1 & 0 & 0 & $\mathbf{0}$ & 1 & 1 \\
2 & 1 & 0 & 1 & 1 & 1 \\
3 & 1 & 0 & 1 & $\mathbf{0}$ & 0 \\
4 & 1 & $\mathbf{0}$ & $\mathbf{0}$ & 0 & 0 \\
\hline
\end{tabular}

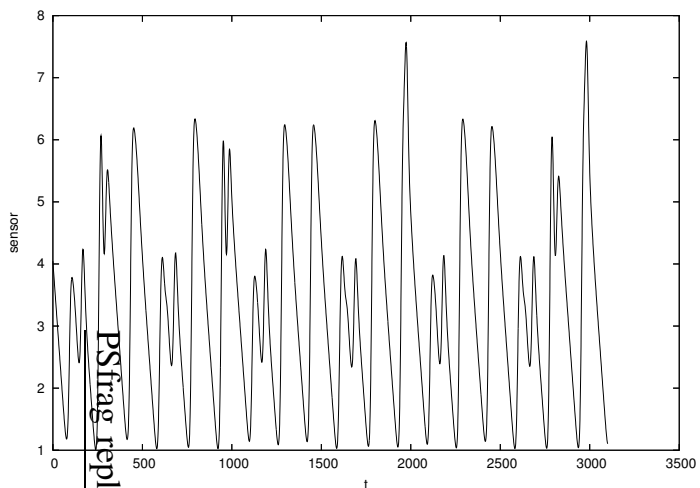

(a)

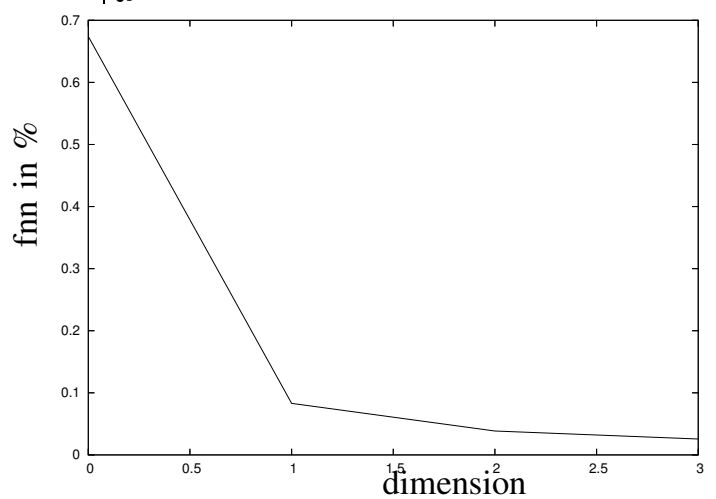

(b)

Fig. 4. High-speed case. (a) Activity of sensor n3, (b) False nearest neighbors

$2 \rightarrow 3$, and its associated crossings whose sum $S L(023)=$ $\sum_{j} \sigma_{j}(023,023)=1+1-2-1=-1$. We compute as well $S L(014)$, and the linking number $L N(023,014)$ between the two orbits. This process must be repeated for every periodic orbit and every couple or periodic orbits, though we only give the results for the couple $(023,014)$ in Tab. II. In the next section we explain how to use those results.

\section{Discussion}

First we must recall that the three dimensional attractor was reconstructed from scalar data recorded by only one range sensor, though a great number of such sensors where available. We notice that this components of the overall phenomenon, 


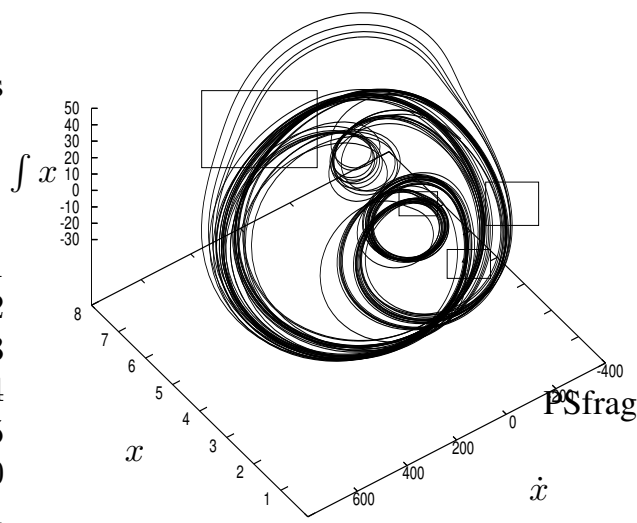

(a) Attractor in differential-integral embedding

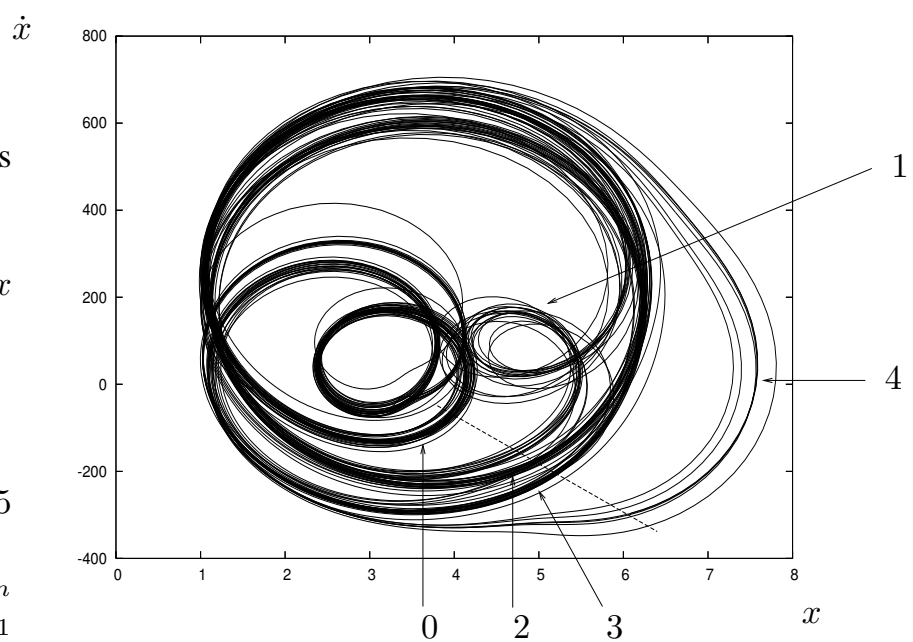

(b) Projection on the plane $x, \dot{x}$

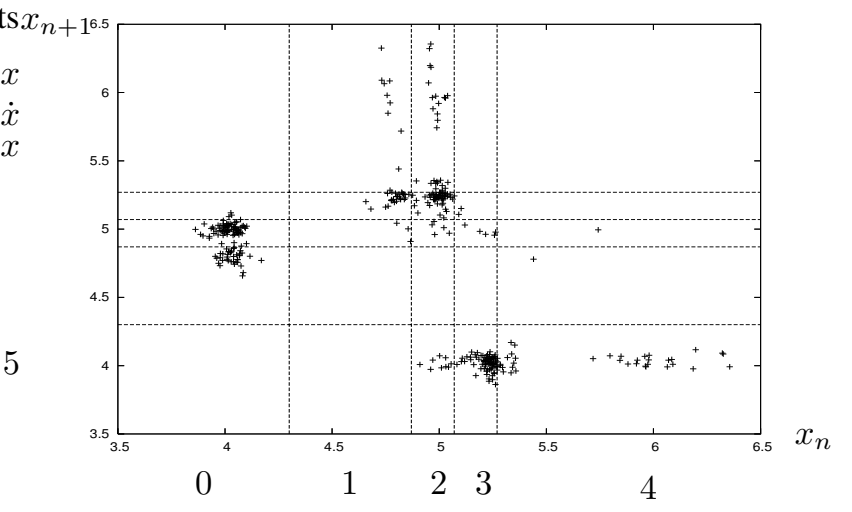

(c) Return map

Fig. 5. (a) Reconstruction of the attractor; (b) Poincaré section represented by the dashed line, the branches are numbered from 0 to 5 and indicated by arrows; (c) Return map from Poincaré section along coordinate $x$. The boxes are numbered from 0 to 4 from the names of the branches.

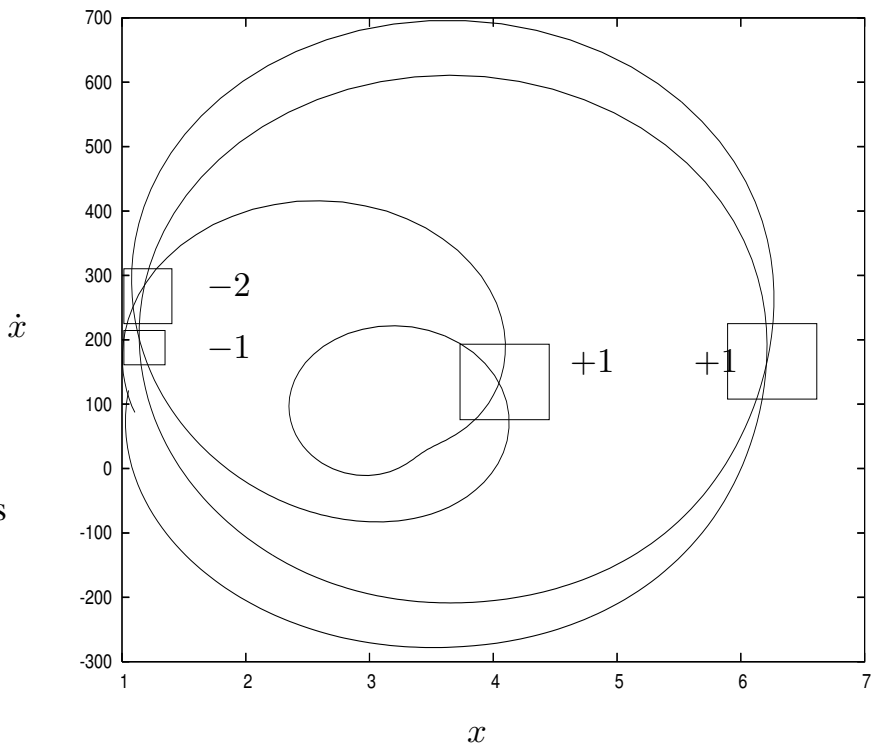

Fig. 6. Projection of periodic orbit $0 \rightarrow 2 \rightarrow 3$ on plane $(x, \dot{x})$. Crossings are enclosed in squares, their crossing number is given.

TABLE II

TOPOLOGICAL INVARIANTS, HIGH SPEED CASE

\begin{tabular}{|c||c|c|}
\hline & 023 & 014 \\
\hline \hline 023 & -1 & -2 \\
014 & -2 & -2 \\
\hline
\end{tabular}

which needs a great number of coupled variables to be completely described, relaxes on a three-dimensional manifold and can be embedded easily using standard techniques.

Secondly we notice -as is exemplified in [20] with other tools- from the presence of stretch and squeeze between branches in the low and high-speed cases that the behavior of the robot presents chaotic properties, even in regular and steady environments with very precise sensors.

Then we proved that it was possible to extract a symbolic encoding form the Poicaré section, that comes from the discrete structure of the attractor (discrete branches, squeeze and stretch lines), even though the exactness of our partition deserves further assessments.

Last we exhibit a part of the unstable periodic orbit spectrum, along with their topological invariants. Comparing the two cases, we see that the attractors share the same basic structure -double loop in the middle, enclosed by a series of wider circles- but observe that in the hi-speed case, two branches were added (smaller loop inside, and outer fringe). The study of orbit forcing and of unfolding is likely to explain such construction and to give predictive insights, but this is left to further developments.

From the point of view of the roboticist, what it the interest of this analysis? It comes from the need to give a quantitative and standardized basis to the description and comparison of all the possible behaviors of a given robot, with a given 
command law, in a given environment. The possible paths to this goal explored up to now, discussed in I, rely on metric considerations: one records a set of trajectories either in sensor space, or in the position space of the robot (e.g. its $x, y$ location in the environment). Then, features can be extracted, a distance is defined on the trajectory or feature space, and a classical classification algorithm partitions the space so as to optimize some quantity (e.g. space occupation). We state that by analogy with Physics, those methods lead to coarse-grained classifications of behaviors, and can be refined. The topological analysis we propose to apply can reveal the inner structure of the dynamics, in a well established and standardized way coming from a branch of Physics dealing with nonlinear phenomena. In spite of their counter-intuitive aspect such tools as the linking numbers given in Tab. II can help comparing and classifying two behaviors in a much sharper way than metric methods do, because they represent in a 1:1 way the structure of the set of trajectories in phase space, made of a set of underlying objects -the unstable periodic orbits- that resist to perturbations (e.g. bifurcations) and organize the geometric object named strange attractor that supports the dynamics. If one can describe this skeleton and its possible modifications under control parameter changes, one can classify precisely the possible behaviors of a robot, and that's what topological invariants do.

\section{CONCLUSION}

In this article we address the problem of classifying the behavior of a robot from topological invariants extracted from recorded sensor data, with no need for a model, and when the robot morphology, the command law, and the environment are likely to change. To do so, we propose an alternative to most existing methods that rely on metric considerations, highly sensitive to perturbations such as noise contamination. This alternative is sought in the field of Physics of Nonlinear systems in chaotic regime (see [2], [6], [18], [9]), that provides analysis schemes able to extract the inner structure of the dynamics, thanks to topological objects such as unstable periodic orbits. At the end of the analysis we are able to associate a group of topological invariants to the robot dynamics, that form a fingerprint of its behavior. From this fingerprint, it is possible to classify different behaviors, in a much sharper way than metric methods do. Next, we plan to cover a larger spectrum of phenomena met in mobile robotics, on the basis of their fingerprints, and to compare quantitatively the discriminative power of this method with metric ones.

\section{REFERENCES}

[1] H.D.I Abarbanel, Analysis of Observed Chaotic Data, Springer, 1995.

[2] D. Auerbach, P. Cvitanović, J.-P. Eckmann, G. Gunaratne, I. Procaccia, Exploring chaotic motion through periodic orbits, Physical Review Letters, vol. 58, No23, 1987.

[3] R.D. Beer, The dynamics of adaptive behavior: A research program, Robotics and Autonomous Systems, Vol.20, No 2, 1997

[4] P. Bergé, Y. Pomeau, C. Vidal, Order within chaos: towards a deterministic approach to turbulence, Wiley, 1986.

[5] J. Borenstein, Y. Koren, The Vector Field Histogram: A Fast ObstacleAvoidance for Mobile Robots, IEEE Journal of Robotics and Automation, Vol. 7, No. 3, pp. 278-288, June 1991.
[6] P. Cvitanović, Periodic orbits as the skeleton of classical and quantum chaos, Physica D, 51, 138-151, 1991.

[7] J.-P. Eckmann, D. Ruelle, Fundamental limitations for estimating dimensions and Lyapunov exponents in dynamical systems, Physica D, 56, 1987.

[8] D.R. Fredkin, J.A. Rice, Method of False Nearest Neighbors: a cautionary note, Physical Review E, Vol. 51, No 4, April 1995.

[9] R. Gilmore, M. Lefranc, The Topology of Chaos, Alice in Stretch and Squeezeland, Wiley, 2002.

[10] B.P. Gerkey, R.T. Vaughan, K. Støy,A. Howard, G.S. Sukhatme, M. Mataric, Most valuable Player: A robot device server for distributed control, Proc. IROS, 2001

[11] B.P. Gerkey, R.T. Howard, A. Howard, The Player/Stage Project: Tools for Multi-Robot and distributed sensor systems, Proc. ICAR 2003.

[12] M. Islam, K. Murase, Chaotic dynamics of a behavior-based miniature mobile robot: effects of environment and control, Neural Networks, 2005.

[13] S. Harnad, The Symbol Grounding Problem, Physica D 42: 335-346, 1990.

[14] R. Hegger, H. Kantz, T. Schreiber, Practical implementation of nonlinear time series methods: The TISEAN package, CHAOS 9, 413, 1999.

[15] K. Han, M. Veloso, Automated robot behavior recognition applied to robotic soccer, Proc. IJCAI-99 Workshop on Team Behaviors and Plan, 1999.

[16] M.B. Kennel, R. Brown, H.D.I. Abarbanel, Determining embedding dimension for phase-space reconstruction using a geometrical construction, Phys. Rev. A, Vol 45 N6, p. 3403, 15 March 1992.

[17] N. Koenig, A. Howard, Design and Use Paradigm for Gazebo, An OpenSource Multi-Robot Simulator, Proc. IROS 2004.

[18] D.P. Lathrop, E.J.Kostelich, Characterization of an experimental strange attractor by periodic orbits, Phys. Rev. A, vol.40, No 7, 1989.

[19] G.M. Mindlin, R. Gilmore Topological analysis and synthesis of chaotic time series, Physica D, 58(1-4), 229-242, 1992.

[20] U. Nehmzow, K. Walker, The behavior or a robot is chaotic, AISB Journal, 2003.

[21] U. Nehmzow, K. Walker, Quantitative description of robot-environment interaction using chaos theory, Robotics and Autonomous Systems, Vol.53, No 3-4, 2005.

[22] M. Ramoni, P. Sebastiani, P. Cohen, Bayesian Clustering by Dynamics, Machine Learning, Vol. 47, No 1, 2002.

[23] Schöner, Dose, Engels, Dynamics of behaior: theory and applications for autonomous robot architectures, Robotics and Autonomous Systems, 1995.

[24] T. Smithers, On quantitative performance measure of robot behaviour, Robotics \& Autonomous Systems, Vol.15, No 1, 1995

[25] M. Wünstel, D. Polani, T. Uthmann, J. Perl, Behavior classification with Self-organizing Maps, Lecture Notes in Computer Science, Vol. 2019, Springer-Verlag, 2001. 